\title{
Adaptive End-to-End QoS Provision for Real-Time Multimedia Applications in Heterogeneous Multi-Hop Wireless Networks
}

\author{
Hong Zhou, Zhongwei Zhang \\ The University of Southern Queensland, Australia \\ Toowoomba QLD 4350, Australia \\ Telephone: +617 4631 1322/5549 \\ Email: hzhou, zhongwei@usq.edu.au
}

\begin{abstract}
A wide range of emerging multimedia services (e.g. IP telephony, video conferencing, video-on-demand) require Quality of Services $(\mathrm{QoS})$ guarantees in both wired and wireless networks. Adaptive QoS provisioning and control are especially necessary to meet the QoS requirements in dynamic heterogeneous multi-hop wireless networks. This paper proposed an adaptative mechanism to achieve better perceptive and quantitative QoS for real-time multimedia services suitable for such networks. The mechanism is built based on the theoretical analysis of the end-to-end delay bounds and the necessary and sufficient conditions to maintain the data flow continuity of real-time connections.
\end{abstract}

\section{Keywords}

adaptive QoS guarantees, delay analysis, multimedia applicaitons

\section{INTRODUCTION}

In recent years, there have been increasing demands for delivering a wide range of real-time multimedia applications such as video conferencing, IP telephony, and video-on-demand over wired and wireless networks. This creates great opportunities as well as challenges for the design and analysis of next-generation heterogeneous wireless networks. All multimedia communications require certain level of Quality of Service guarantees (QoS). Future networks including mobile ad hoc networks, cellular networks, and wireless sensor networks are required to support realtime multimedia applications with Quality of Service (QoS) guarantees.

Permission to make digital or hard copies of all or part of this work for personal or classroom use is granted without fee provided that copies are not made or distributed for profit or commercial advantage and that copies bear this notice and the full citation on the first page. To copy otherwise, or republish, to post on servers or to redistribute to lists, requires prior specific permission and/or a fee.

Mobimedia'09, September 7-9, 2009, London, UK.

Copyright 2009 ICST 978-963-9799-62-2/00/0004... \$5.00
Scheduling algorithms play a key role in satisfying the delay requirements. In the past twenty years, a significant volume of research has been published in literature on scheduling algorithms such as Packet-by-packet Generalized Processor Sharing (PGPS) [1], Self-Clocked Fair Queueing (SCFQ) [2], Latency-Rate (LR) Server [3], Start-time Fair Queueing (SFQ) [4], Queueing Latency-Rate Servers [8], Wireless Packet Scheduling (WPS) [5] and Energy Efficient Weighted Fair Queueing ( $E^{2}$ WFQ) [6]. The general delay analysis of scheduling algorithms (QLR servers) in multi-hop networks have been addressed in [8]. The end-to-end delay is simply the sum of three delay components, namely, the smoothing delay, the multiplexing delay and the transmission delay. The smoothing delay depends on the burstiness of the traffic; the multiplexing delay is determined by the scheduling algorithms employed in the router and competing background traffic; the transmission delay is simply the function of link speed and packet size. Each component is of different nature and different approaches are needed to quantify each component [8]. Unlike non-real-time data services, the continuity of real-time data flow needs to be maintained to provide satisfactory real-time services. Buffers are used at individual network nodes especially at the destination end. Whenever buffer overflows or underflows, the continuity of the data flow breaks down and causes the QoS degradation. This paper proposes an end-to-end delay-based adaptive QoS provisioning mechanism to achieve better data flow continuity for real-time multimedia services in heterogeneous multi-hop networks.

The rest of this paper is organised as follows. In Section 2, a general discrete-time queueing system model is defined and illustrated. In Section 3, the end-to-end delay bounds in terms of three delay components is provided. The method is suitable for any packet-switched network with arbitrary topology. In Section 4, the conditions under which the real-time data flow continuity is preserved are examined and an adaptive method for improve QoS is provided. Finally, in Section 5 we conclude the paper.

\section{PRELIMINARY CONCEPTS}

A general queueing system which models all packet-level schedulers is illustrated in Figure 1. In Figure 1, a general queue server 